\title{
Bandwidth statistics from the eigenvalue moments for the Harper-Hofstadter problem
}

\author{
O Lipan \\ Division of Physics, Mathematics, and Astronomy, California Institute of Technology, Pasadena, \\ CA 91125, USA \\ E-mail: olipan@its.caltech.edu
}

Received 24 January 2000, in final form 12 July 2000

\begin{abstract}
A method for studying the product of bandwidths for the Harper-Hofstader model is proposed, which requires knowledge of the moments of the midband energies. A general formula for these moments is conjectured, and the asymptotic representation for the product of bandwidths computed in the limit of a weak magnetic flux using Szegö's theorem for Hankel matrices. Then a first approximation for the edge of the butterfly spectrum is given and its connection with Lévy's formula for Brownian motion discussed.
\end{abstract}

\section{Introduction}

A system of electrons on a square lattice in a uniform magnetic field displays an intricate energy spectrum. For a rational magnetic flux, i.e. $\Phi=2 \pi p / q$, with $p$ and $q$ relatively prime integers, there are $q$ bands in the spectrum if $q$ is odd. For $q$ even, the bands at $E=0$ are touching $[6,7]$. The aim of this paper is to study the asymptotic properties of the product of bandwidths. First, a method to obtain the band spectrum is discussed. Secondly, the problem to be addressed is defined more precisely. The Hamiltonian of electrons on a square lattice in a uniform magnetic field, in terms of magnetic translations $T_{1}$ and $T_{2}$, is given by

$$
H=T_{1}+T_{1}^{*}+T_{2}+T_{2}^{*}
$$

where $T_{1}$ and $T_{2}$ obeys the following commutation relation:

$$
T_{1} T_{2}=\mathrm{e}^{\mathrm{i} \Phi} T_{2} T_{1} .
$$

In (1.2), $\Phi$ is the magnetic flux (see [26,30] for details concerning magnetic translations).

For a rational magnetic flux,

$$
\Phi=2 \pi \frac{p}{q}
$$

the Hamiltonian becomes a $q \times q$ matrix. From now on, $p$ and $q$ will be relatively prime integers.

One representation for the translation operators is

$$
T_{1}=\mathrm{e}^{\mathrm{i} \theta_{1}} w_{1} \quad T_{2}=\mathrm{e}^{\mathrm{i} \theta_{2}} w_{2}
$$


where

$$
\begin{aligned}
& w_{1}=\left(\begin{array}{ccccc}
0 & 1 & 0 & \cdots & 0 \\
& \ddots & \ddots & \ddots & \\
\vdots & & \ddots & \ddots & 0 \\
& & & \ddots & 1 \\
1 & & \ldots & & 0
\end{array}\right) \\
& w_{2}=\operatorname{diag}\left(\mathrm{e}^{\mathrm{i} \Phi}, \mathrm{e}^{\mathrm{i} 2 \Phi}, \ldots, \mathrm{e}^{\mathrm{i} q \Phi}\right)
\end{aligned}
$$

and $\theta_{j}, j=1,2$ are real numbers (Bloch parameters).

In this representation, the Hamiltonian is represented by the following matrix:

$$
H(\theta)=\left(\begin{array}{ccccc}
a_{1} & b_{1} & 0 & \ldots & \bar{b}_{q} \\
\bar{b}_{1} & a_{2} & b_{2} & \ldots & \\
0 & \bar{b}_{2} & a_{3} & \ddots & \\
\vdots & \vdots & \ddots & \ddots & b_{q-1} \\
b_{q} & & 0 & \bar{b}_{q-1} & a_{q}
\end{array}\right)
$$

where

$$
\begin{aligned}
& a_{n}(\theta)=2 \lambda \cos \left(\Phi n+\theta_{2}\right) \quad(n=1,2, \ldots, q) \\
& b_{n}(\theta)=\mathrm{e}^{\mathrm{i} \theta_{1}}
\end{aligned}
$$

and $\theta=\left(\theta_{1}, \theta_{2}\right)$.

Chamber's relation [10], states that the characteristic polynomial of the Hamiltonian matrix decomposes into a $\theta$-independent polynomial $P_{p / q}(E)$ of degree $q$ and a function $h(\theta)$,

$$
\begin{aligned}
& \operatorname{det}(H(\theta)-E)=P_{p / q}(E)+h(\theta) \\
& h(\theta)=(-1)^{q-1} 2\left(\cos \left(q \theta_{1}\right)+\cos \left(q \theta_{2}\right)\right) .
\end{aligned}
$$

When $\theta$ varies, the function $h(\theta)$ varies between -4 and 4 , so, for a given rational flux $\Phi=2 \pi p / q$, the bands are obtained by intercepting the graph of the polynomial $P_{p / q}(E)$ with the horizontal lines drawn at 4 and -4 . Figure 1 shows the procedure for $p=1, q=3$.

For the case in figure 1 there are three bands, because $q=3$. Counting from the left these are Band $_{1}=[-1-\sqrt{3},-2]$, Band $_{2}=[1-\sqrt{3},-1+\sqrt{3}]$ and Band $3=[2,1+\sqrt{3}]$. Denote the widths of these bands by $\Delta_{1}, \Delta_{2}$ and $\Delta_{3}$, respectively. In general, for a polynomial $P_{p / q}(E)$, the widths will be denoted by, counting from negative to positive energies, $\Delta_{1}, \ldots, \Delta_{q}$. The roots of the polynomial $P_{p / q}(E)$ are eigenvalues for the Hamiltonian, for special values of Bloch momenta $\theta=(\pi / q, \pi / q)$. Let us call these roots $E_{1}, \ldots, E_{q}$, counting from the negative to positive values of $E$. For the example in figure $1, p=1, q=3$, these eigenvalues are $E_{1}=-\sqrt{6}, E_{2}=0, E_{3}=\sqrt{6}$. The energies $E_{1}, E_{2}, \ldots, E_{q}$ are called midband energies. These energies were studied in $[2,30]$ using the Bethe ansatz. The problem can be now stated as follows: find the asymptotic formula for the products of the bandwidths, i.e.

$$
\prod_{n=1}^{q} \Delta_{n} .
$$




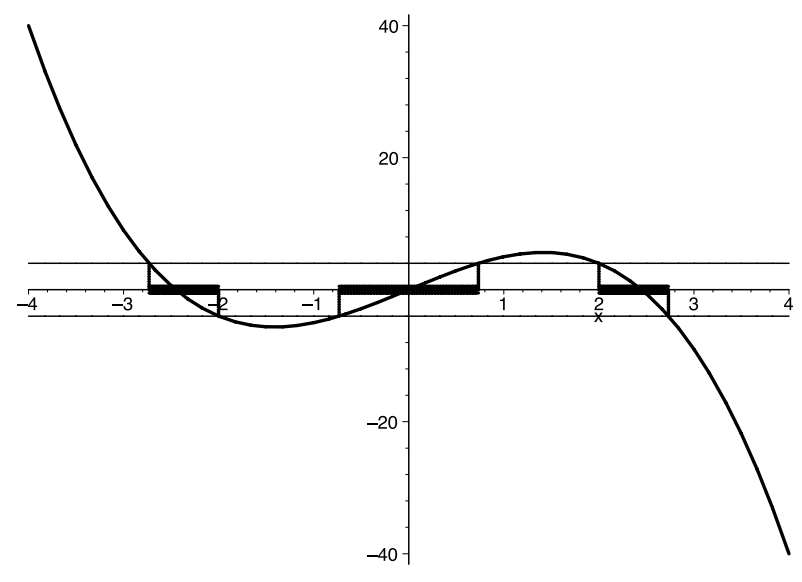

Figure 1. Bands for $P_{1 / 3}=-x^{3}+6 x$.

In the final section of the paper, it is found that, for a weak magnetic field $\Phi=2 \pi \frac{1}{q} \rightarrow 0$, the asymptotic formula can be arranged as a product of terms, each term approaching zero faster than the next-neighbour term, equation (5.14):

$$
\prod_{n=1}^{q} \Delta_{n} \sim 2^{-q^{2}} q^{-q} \exp \left(-q d_{0}+d_{1}+\mathrm{O}(1)\right) .
$$

Here, $d_{0}$ and $d_{1}$ are some constants. Before ending this section, we write the polynomial $P_{p / q}(E)$ as a characteristic polynomial of a tridiagonal matrix. In this way it will become easier to compute the moments of the roots of the polynomial $P_{p / q}(E)$, which we need to obtain the asymptotic formula (1.11). The moments will be the main object of study in section 3. From this point to the end of the introduction, the formulae are taken from [20].

The roots of $P_{p / q}(E)$ are special eigenvalues and correspond for those $\theta$ for which $h(\theta)=$ 0 . Studying the polynomial $P_{p / q}(E)$ becomes easier if we choose another representation for the Hamiltonian matrix:

$$
\begin{aligned}
& T_{1}=\mathrm{e}^{\mathrm{i} \theta_{1}} w_{1} \\
& T_{2}=\mathrm{e}^{\mathrm{i} \Phi / 2} \mathrm{e}^{\mathrm{i} \theta_{2}} \mathrm{e}^{\mathrm{i} \theta_{1}} w_{2} w_{1} .
\end{aligned}
$$

In this case the Hamiltonian matrix acquires the form

$$
\tilde{H}(\theta)=\left(\begin{array}{ccccc}
0 & a_{1} & 0 & \ldots & \bar{a}_{q} \\
\bar{a}_{1} & 0 & a_{2} & \ldots & \\
0 & \bar{a}_{2} & 0 & \ddots & 0 \\
\vdots & \vdots & \ddots & \ddots & a_{q-1} \\
a_{q} & & 0 & \bar{a}_{q-1} & 0
\end{array}\right)
$$

with

$$
a_{r}=\left(1+\exp \left[\mathrm{i} \Phi\left(r+\frac{1}{2}\right)+\mathrm{i} \theta_{2}\right]\right) \mathrm{e}^{\mathrm{i} \theta_{1}} .
$$

Chamber's relation in the above representation is

$$
\operatorname{det}(\tilde{H}(\theta)-E)=P_{p / q}(E)+\tilde{h}(\theta)
$$


where

$$
\tilde{h}(\theta)=(-1)^{q-1} 2\left(\cos \left(q \theta_{1}\right)+(-1)^{p} \cos \left(q\left(\theta_{2}+\theta_{1}\right)\right)\right) .
$$

This last representation is convenient because for the special value $\theta_{0}=\left(\theta_{10}, \theta_{20}\right)=$ $(0, \pi(1+p / q))$, simultaneously

$$
\tilde{h}\left(\theta_{0}\right)=0
$$

and

$$
a_{q}\left(\theta_{0}\right)=0
$$

which makes $\tilde{H}\left(\theta_{0}\right)$ a tridiagonal matrix. Out of this tridiagonal matrix, recursive equations can be deduced for generating the polynomial $P_{p / q}(E)$. Let $\tilde{P}_{0}(E), \tilde{P}_{1}(E), \ldots, \tilde{P}_{q}(E)$ be a sequence of polynomials generated by the following relations:

$$
\begin{aligned}
\tilde{P}_{0}(E) & =1 \\
\tilde{P}_{1}(E) & =-E \\
\tilde{P}_{n}(E) & =-E \tilde{P}_{n-1}(E)-\beta_{n-1} \tilde{P}_{n-2}(E) \quad(n=2,3, \ldots, q)
\end{aligned}
$$

where

$$
\begin{aligned}
& \beta_{n}=4 \sin ^{2}\left(\frac{\Phi}{2} n\right) \quad(n=1,2, \ldots, q) \\
& \Phi=2 \pi p / q .
\end{aligned}
$$

It follows that $\tilde{P}_{q}(E)=P_{p / q}(E)$ and that the polynomial $P_{p / q}(E)$ is the characteristic polynomial of the following tridiagonal matrix, which contains only positive numbers:

$$
\begin{aligned}
& M=\left(\begin{array}{ccccc}
0 & \beta_{1} & 0 & \cdots & 0 \\
1 & 0 & \beta_{2} & \cdots & \\
0 & 1 & 0 & \ddots & 0 \\
\vdots & \vdots & \ddots & \ddots & \beta_{q-1} \\
0 & & 0 & 1 & 0
\end{array}\right) \\
& P_{p / q}(E)=\operatorname{det}(M-E) .
\end{aligned}
$$

\section{Bandwidth product}

From the paper [21], it is known that the bandwidths obey the inequalities (with e $=\exp (1)=$ $2.718 \ldots$..

$$
\frac{2(1+\sqrt{5})}{\left|P_{p / q}^{\prime}\left(E_{n}\right)\right|}<\Delta_{n}<\frac{8 \mathrm{e}}{\left|P_{p / q}^{\prime}\left(E_{n}\right)\right|} \quad n=1, \ldots, q
$$

where $P_{p / q}^{\prime}\left(E_{n}\right)$ is the derivative of $P_{p / q}(E)$ computed at the eigenvalue $E_{n}$, which are the roots of $P_{p / q}(E)$. Consequently,

$$
(2+2 \sqrt{5})^{q} \frac{1}{\left|\prod_{n=1}^{q} P_{p / q}^{\prime}\left(E_{n}\right)\right|}<\prod_{n=1}^{q} \Delta_{n}<(8 \mathrm{e})^{q} \frac{1}{\left|\prod_{n=1}^{q} P_{p / q}^{\prime}\left(E_{n}\right)\right|}
$$


The product $\prod_{n=1}^{q} P_{p / q}^{\prime}\left(E_{n}\right)$ can be re-expressed as

$$
\prod_{j>i=1}^{q}\left(E_{j}-E_{i}\right)^{2}
$$

The formula (2.3) can be written as a product of two Vandermonde determinants:

$$
\prod_{j>i=1}^{q}\left(E_{j}-E_{i}\right)^{2}=\left|\begin{array}{cccc}
1 & 1 & \cdots & 1 \\
E_{1} & E_{2} & \cdots & E_{q} \\
E_{1}^{2} & E_{2}^{2} & \cdots & E_{q}^{2} \\
\vdots & \vdots & & \vdots \\
E_{1}^{q-1} & E_{2}^{q-1} & \cdots & E_{q}^{q-1}
\end{array}\right|\left|\begin{array}{ccccc}
1 & E_{1} & E_{1}^{2} & \cdots & E_{1}^{q-1} \\
1 & E_{2} & E_{2}^{2} & \cdots & E_{2}^{q-1} \\
\vdots & \vdots & \vdots & & \vdots \\
1 & E_{q} & E_{q}^{2} & \cdots & E_{q}^{q-1}
\end{array}\right| .
$$

By this observation, the product $\prod_{n=1}^{q} P_{p / q}^{\prime}\left(E_{n}\right)$ can be written in terms of the eigenvalue moments, defined as

$$
s_{2 k}=\sum_{n=1}^{q} E_{n}^{2 k} \quad k=1,2,3, \ldots
$$

Putting it all together gives

$$
(2+2 \sqrt{5})^{q} \frac{1}{\sigma_{q-1}}<\prod_{n=1}^{q} \Delta_{n}<(8 \mathrm{e})^{q} \frac{1}{\sigma_{q-1}}
$$

where $\sigma_{q-1}$ is the Hankel determinant:

$$
\sigma_{q-1}=\left|\begin{array}{ccccc}
q & s_{1} & s_{2} & \cdots & s_{q-1} \\
s_{1} & s_{2} & s_{3} & \cdots & s_{q} \\
s_{2} & s_{3} & s_{4} & \cdots & s_{q+1} \\
\vdots & \vdots & \vdots & & \vdots \\
s_{q-1} & s_{q} & s_{q+1} & \cdots & s_{2 q-2}
\end{array}\right|
$$

To obtain the asymptotic representation of the product of the bandwidths, as $q \rightarrow \infty$, a separate study of the eigenvalue moments, $s_{2 k}$, is necessary. The next section is dedicated to this study.

\section{Eigenvalue moments}

First, we show that

$$
s_{2 k}=q \sum_{j=0}^{\left[k^{2} / 4\right]} a_{j}(k) \cos (2 \pi j p / q) \quad \text { if } \quad q>k
$$

where $a_{j}$ are integer numbers that do not depend on $p$ and $q$. Here $\left[k^{2} / 4\right]$ is the integer part of $k^{2} / 4$.

To see this, note that the eigenvalue moments can be computed from the trace of the powers of the matrix $M$, equation (1.23):

$$
s_{2 k}=\operatorname{Tr}\left(M^{2 k}\right) .
$$


Let us consider two examples, for $k=4$ and 6:

$$
\begin{aligned}
& \operatorname{Tr}\left(M^{4}\right)=\sum_{m=1}^{q-1}\left(2 \beta_{m}^{2}+4 \beta_{m-1} \beta_{m}\right) \\
& \operatorname{Tr}\left(M^{6}\right)=\sum_{m=1}^{q-1}\left(6 \beta_{m}^{3}+6 \beta_{m-1}^{2} \beta_{m}+6 \beta_{m-1} \beta_{m}^{2}+6 \beta_{m-2} \beta_{m-1} \beta_{m}\right)
\end{aligned}
$$

where $\beta_{m}=0$ if $m<0$. The sum can be extended to $q$, because $\beta_{q}=0$. We find then (here $\left.C:=\mathrm{e}^{2 \pi \mathrm{i} p / q}\right)$ :

$$
\begin{aligned}
s_{4}=\operatorname{Tr}\left(M^{4}\right) & =\sum_{m=1}^{q}\left(2\left(2-C^{m}-C^{-m}\right)^{2}+4\left(2-C^{m-1}-C^{-m+1}\right)\left(2-C^{m}-C^{-m}\right)\right) \\
& =4 q(7+2 \cos (2 \pi p / q)) .
\end{aligned}
$$

In general,

$$
s_{2 k}=\sum_{m=1}^{q} \sum_{l_{0}+l_{1}+\cdots+l_{j}=k} d_{l_{0} l_{1} \cdots l_{j}} \beta_{m-j}^{l_{j}} \cdots \beta_{m-1}^{l_{1}} \beta_{m}^{l_{0}}
$$

where $j$ takes values between 0 and $k-1$; compare with (3.4). The coefficients $d_{l_{0} l_{1} \cdots l_{j}}$ do not depend on $p$ and $q$. They only depend on $k$ and on $\left(l_{0}, l_{1}, \ldots, l_{j}\right)$. Moreover, we can write

$$
\sum_{m=1}^{q} \beta_{m-j}^{l_{j}} \cdots \beta_{m-1}^{l_{1}} \beta_{m}^{l_{0}}=\sum_{n} g_{n} C^{\xi_{n}}
$$

where $g_{n}$ are integer numbers which do not depend on $p$ and $q$ and the index $n$ runs from 0 over a finite range. In the above sum, the only non-zero terms will arise for those powers $\xi_{n}$ which do not depend on $m$. This fact is a consequence of the structure of $\left(l_{0}, l_{1}, \ldots, l_{j}\right)$ and not of $p$ and $q$. The sum over $m$ will collect $q$ identical terms so in the result the parameter $q$ will be factor out. Note that for a fixed $k$ the same $\left(l_{0}, l_{1}, \ldots, l_{j}\right)$-structure is present for all $q>k$. For $q \leqslant k$, some $\left(l_{0}, l_{1}, \ldots, l_{j}\right)$ are not present. For example, for $q=k$ the term $\beta_{m-k+1} \cdots \beta_{m-1} \beta_{m}$ is not present, whereas for all $q>k$ it is present. The maximum value for $j$ in (3.1) is $\left[k^{2} / 4\right]$. This will be explained later, in section 4 , after formula (4.5).

To proceed further, note that in the formula for $s_{2 k}$, equation (3.1), the parameter $p$ appears only in the ratio $p / q$. Because of this, the analysis of the eigenvalue moments can be extended to all real magnetic fluxes. Indeed, for an irrational $\omega$, let us consider a sequence of rational approximants $p_{n} / q_{n} \rightarrow \omega$, as $n \rightarrow \infty$. For each $n$ we obtain

$$
s_{4, n}=4 q_{n}\left(7+2 \cos \left(2 \pi p_{n} / q_{n}\right)\right) .
$$

So, in the limit $n \rightarrow \infty$,

$$
\left\langle E^{4}(\omega)\right\rangle:=\lim _{n \rightarrow \infty} \frac{\sum_{j=1}^{q_{n}} E_{j, n}^{4}}{q_{n}}=4(7+2 \cos (2 \pi \omega))
$$


where $E_{j, n}, j=1, \ldots, q_{n}$ are the special eigenvalues (roots of $P_{p_{n} / q_{n}}(E)$ ) for the flux $\Phi_{n}=2 \pi p_{n} / q_{n}$. Here are the first five limit eigenvalue averages:

$$
\begin{aligned}
& \left\langle E^{2}(\omega)\right\rangle=4 \\
& \left\langle E^{4}(\omega)\right\rangle=4(7+2 \cos (2 \pi \omega)) \\
& \left\langle E^{6}(\omega)\right\rangle=4(58+36 \cos (2 \pi \omega)+6 \cos (4 \pi \omega)) \\
& \left\langle E^{8}(\omega)\right\rangle=4(539+504 \cos (2 \pi \omega)+154 \cos (4 \pi \omega)+24 \cos (6 \pi \omega)+4 \cos (8 \pi \omega)) \\
& \left\langle E^{10}(\omega)\right\rangle=4(5486+6580 \cos (2 \pi \omega)+2770 \cos (4 \pi \omega)+780 \cos (6 \pi \omega) \\
& \quad+210 \cos (8 \pi \omega)+40 \cos (10 \pi \omega)+10 \cos (12 \pi \omega)) .
\end{aligned}
$$

Now, a natural question comes to mind: is there a general formula for $s_{2 k}$, or equivalently for $\left\langle E^{2 k}(\omega)\right\rangle$ ?

At this time, I have a partial answer to this question. From the first eigenvalue moments the following statement can be inferred:

$$
\left\langle E^{2 k}(\omega)\right\rangle=\left(\begin{array}{c}
2 k \\
k
\end{array}\right)^{2}\left(1+\sum_{m=1}^{\left[k^{2} / 4\right]} k \frac{(2 k-2 m-1) ! !}{(2 k-1) ! !} \frac{k !}{\left(k-j_{m}-2\right) !} P_{m}(k) \sin ^{2 m}(\pi \omega)\right)
$$

where for each $m$ the positive integer number $j_{m}$ is that one for which

$$
2\left[\left(j_{m}+1\right)^{2} / 4\right]+2 \leqslant 2 m \leqslant 2\left[\left(j_{m}+2\right)^{2} / 4\right] .
$$

The first pairs $\left(2 m, j_{m}\right)$ are $(2,0),(4,1),(6,2),(8,2),(10,3),(12,3),(14,4),(16,4),(18,4)$ $P_{m}(k)$ is a polynomial of degree $3 m-j_{m}-3$. This is so because the power of $k$ in front of $\sin ^{2 m}(\pi p / q)$ is $2 m$. There is one more detail about $P_{m}(k)$ which can be found by inspection of the first few terms. Namely, the coefficients of the largest power in $k$, i.e. $\lambda_{m}$ in

$$
P_{m}(k)=\lambda_{m} k^{3 m-j_{m}-3}+\cdots
$$

are generated by

$$
\frac{\sqrt{2} x}{\sinh (\sqrt{2} x)}=\sum_{m=0}^{\infty} \lambda_{m} x^{2 m}
$$

The following is a list of the first three polynomials $P_{m}$ (the first eight polynomials are listed in the appendix):

$$
\begin{aligned}
& P_{1}(k)=-\frac{1}{3} \\
& P_{2}(k)=\frac{1}{90}\left(7 k^{2}-4 k-15\right) \\
& P_{3}(k)=-\frac{1}{1890}\left(31 k^{4}-60 k^{3}-101 k^{2}+66 k+280\right) .
\end{aligned}
$$

From the formula (3.15) we find $s_{2 k}$ that we need in (2.7). Namely,

$$
s_{2 k}=q\left\langle E^{2 k}(p / q)\right\rangle \quad \text { if } \quad q>k .
$$

In order to compute the asymptotic representation of the Hankel determinant (2.7), knowledge of $P_{m}(k)$ will suffice, because the largest moment needed in (2.7) is $s_{2 q-2}$ which is still given by the formula (3.22), since $q>(2 q-2) / 2$. Because there is no general formula for the 
polynomials $P_{m}(k)$, the asymptotic representation for the bandwidth product for all magnetic fluxes cannot be computed.

Still, the case $\Phi=2 \pi \frac{1}{q}$ when $q \rightarrow \infty$ can be analysed, which is done in the final section. Before that, the eigenvalue moments can be further studied, using the summation formula (3.17). The analysis from the next section, besides its intrinsic value, is also useful for understanding the asymptotic formula for the bandwidth product in the case of a weak magnetic flux.

\section{The edge of the butterfly spectrum and Lévy's formula for Brownian motion}

In order to obtain a first approximation for the edge of the spectrum, we can make use of the general formula which gives the eigenvalue moments (3.15). Let us write the formula for the eigenvalue moments as a power series in $k$ and retain only the largest power in $k$. Call the result the first approximation to the eigenvalue moments, and use (1) as a superscript to mark it:

$$
\sum_{j=1}^{q_{n}} E_{j, n}^{2 k,(1)}=q_{n}\left(\begin{array}{c}
2 k \\
k
\end{array}\right) \sum_{m=0}^{2\left[k^{2} / 4\right]} \lambda_{m} \frac{1}{2^{m}} k^{2 m} \sin ^{2 m}\left(\pi \frac{p_{n}}{q_{n}}\right) .
$$

For $k \rightarrow \infty$ we obtain

$$
\sum_{j=1}^{q_{n}} E_{j, n}^{2 k,(1)}=q_{n}\left(C_{2 k}^{k}\right)^{2} \frac{k \sin \left(\pi\left(p_{n} / q_{n}\right)\right)}{\sinh \left(k \sin \left(\pi\left(p_{n} / q_{n}\right)\right)\right)}
$$

and

$$
E_{\max }^{(1)}(\omega)=\lim _{p_{n} / q_{n} \rightarrow \omega} \lim _{k \rightarrow \infty}\left(q_{n}\left(C_{2 k}^{k}\right)^{2} \frac{k \sin \left(\pi\left(p_{n} / q_{n}\right)\right)}{\sinh \left(k \sin \left(\pi\left(p_{n} / q_{n}\right)\right)\right)}\right)^{1 / 2 k}
$$

from which, the first approximation of the edge of the butterfly spectrum is given by

$$
E_{\max }^{(1)}(\omega)=4 \mathrm{e}^{-\frac{1}{2} \sin (\pi \omega)}
$$

where $0 \leqslant \omega \leqslant 1$ and the flux is $\Phi=2 \pi \omega$.

Figure 2 presents, for each $p / q, q=53, p=1,2, \ldots, 52$, the spectrum $E_{j}(p / q)$, $j=1, \ldots, q$ on a horizontal line. The vertical coordinate is the flux (though we represented only $p / q$ and not $2 \pi p / q)$. The horizontal coordinate is obviously energy. Superimposed on

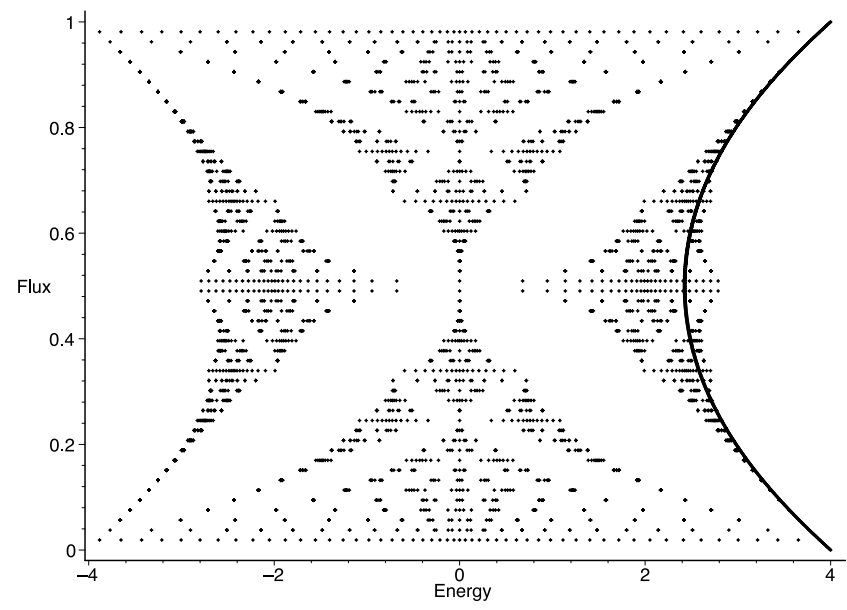

Figure 2. Spectrum for $q=53$ and the function $4 \mathrm{e}^{-\frac{1}{2} \sin (\pi \omega)}$, the first approximation for the edge. 
the above-described spectrum is the graph of $4 \mathrm{e}^{-\frac{1}{2} \sin (\pi \omega)}$ as a function of $\omega$, which is the first approximation to the edge of the spectrum. Second-order approximations can be obtained by summing the next largest power of $k$, namely all terms that contain $k^{2 m-1}$. It is quite clear that a full understanding of the sequence of polynomials $P_{m}(k)$ will enable us to decipher the fractal character of the edge of the spectrum.

At this point it is worth mentioning works connected to the eigenvalue moments formula, (3.15). The geometric interpretation of the trace of the Hamiltonian can be found, for example, in [8]. For this, consider the square lattice $Z^{2}$, where $Z$ are the integer numbers. Let $\Gamma_{2 k}$ be the set of all paths of lengths $2 k$ which start and end at the origin $(0,0)$. For such a path $\Gamma$, let $\operatorname{area}(\Gamma)$ be the oriented area enclosed by $\Gamma$ (see [25] for details). Then we have

$$
\operatorname{Tr}\left(H^{2 k}\right)=q \sum_{\Gamma \in \Gamma_{2 k}} \mathrm{e}^{\mathrm{i} \pi \frac{p}{q} \operatorname{area}(\Gamma)} .
$$

Compare this expression with the formulae for the eigenvalue moments. For example, for $k=2$ we obtain that $4 \times 7=28$ represents the number of paths of length 4 starting and ending at the origin, which enclose zero area. With the interpretation (4.5) for the eigenvalue moments, it is easy to explain the maximum value $\left[k^{2} / 4\right]$ reached by $j$ in (3.1). The maximum value for the $\operatorname{area}(\Gamma)$, when $\Gamma$ has a fixed perimeter of $2 k$, is achieved when $\Gamma$ is a square with edge of length $k / 2$ (when $k$ is even), so the area is $k^{2} / 4$. By the same token, for $k$ odd, the maximum area is $\left[k^{2} / 4\right]$.

As $k \rightarrow \infty$ random walks with $2 k$ steps, approach (using a suitable renormalization, see [9]) two-dimensional Brownian motion. The probability distribution of the areas enclosed by a planar Brownian motion was computed first by Lévy [24]. Lévy’s result is

$$
E[\exp (\mathrm{i} g \text { area })]=\frac{g \text { area } / 2}{\sinh (g \text { area } / 2)}
$$

where $E$ [ ] is the expectation value and $g$ is a real parameter. Also, in a recent paper [25], Mingo and Nica studied the power sums of the areas. What these authors found is that

$$
\left(\begin{array}{c}
2 k \\
k
\end{array}\right)^{-1} \sum_{\Gamma \in \Gamma_{2 k}}(\operatorname{area}(\Gamma))^{2 m}=R_{2 m}(k) \quad \text { if } \quad k>2 m
$$

where $R_{2 m}(k)$ is a rational function in $k$. The degree of $R_{2 m}(k)$ (i.e. the difference between the degrees of the numerator and the denominator of $R_{2 m}(k)$ ) is equal to $2 m$, and the leading coefficient of $R_{2 m}(k)$, call it $\nu_{2 m}$, is generated by

$$
\frac{z}{\sin z}=\sum_{j=0}^{\infty} \frac{v_{2 m}}{(2 m) !}(2 z)^{2 m}
$$

Both of these results are connected with the generating function for the leading coefficients of the polynomials $P_{m}(k)$, equation (3.18). The formula for the eigenvalue moments, equation (3.15) goes beyond Lévy's formula. From this perspective, a full understanding of the polynomials $P_{m}(k)$ will shed new light on planar Brownian motion.

\section{Asymptotic representation of the product of bandwidths for a weak magnetic field and Szegö's theorem}

We now aim to compute the asymptotic representation of the product of the bandwidths, $\prod_{n=1}^{q} \Delta_{n}$, for a weak magnetic flux, $\Phi=2 \pi \frac{1}{q}$, when $q \rightarrow \infty$. We will use the inequality (2.6) together with the asymptotic representation of the Hankel determinant, (2.7). The entries of 
the Hankel determinant are the moments $s_{2 k}$, see (3.22). Up to the first order of approximation, equation (4.2), the moments are

$$
s_{2 k}=q\left(\begin{array}{c}
2 k \\
k
\end{array}\right)^{2} \frac{k \sin (\pi / q)}{\sinh (k \sin (\pi / q))} .
$$

To go further, and because of lack of knowledge of the polynomials $P_{m}(k)$, it is assumed that (for the case of a weak flux), the second and all other orders of approximation are much smaller than the first order. Moreover, because

$$
C_{1}<\frac{k \sin (\pi / q)}{\sinh (k \sin (\pi / q))} \mathrm{e}^{k / 2 q}<C_{2} \quad k=1,2, \ldots, q-1
$$

the influence of the first approximation term, i.e.

$$
\frac{k \sin (\pi / q)}{\sinh (k \sin (\pi / q))}
$$

on the asymptotic formula is considered to be of the same order of magnitude as given by the term $\mathrm{e}^{-k / 2 q}$. In (5.2) $C_{1} \sim \pi \mathrm{e} / \sinh (\pi)=0.739$ and $C_{2} \sim \mathrm{e}^{1 / \pi} / \sinh (1)=1.169$. Thus the moments $s_{2 k}$ to be used to find the asymptotic formula for the Hankel determinant are

$$
s_{2 k}=q\left(\begin{array}{c}
2 k \\
k
\end{array}\right)^{2} \mathrm{e}^{-k / 2 q}
$$

and it follows that

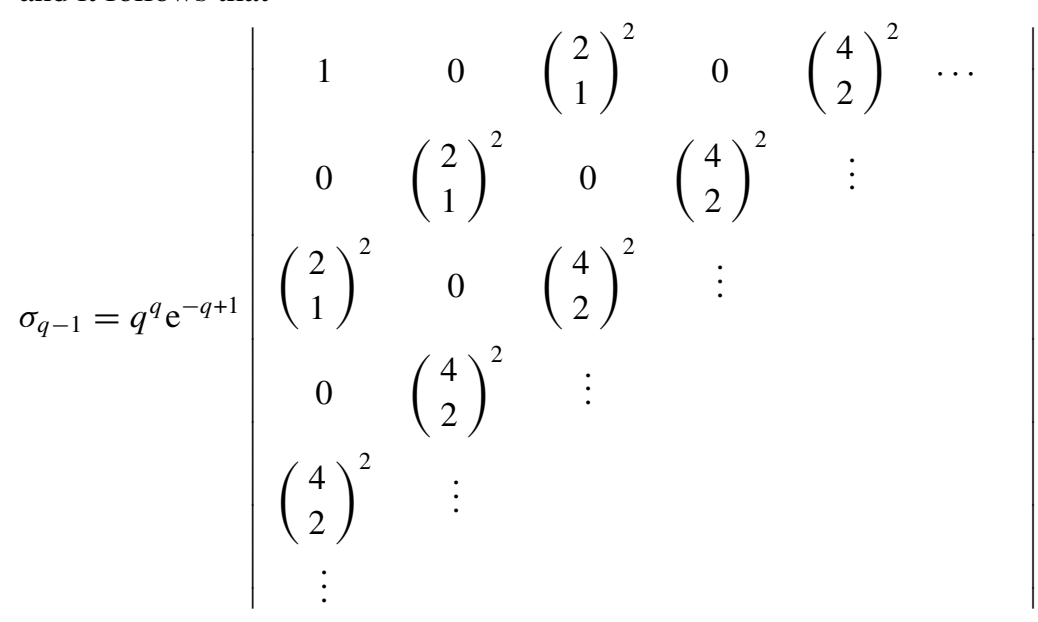

The dimension of $\sigma_{q-1}$ is $q$. To find the asymptotic representation of $\sigma_{q-1}$, as $q \rightarrow \infty$, we shall employ a theorem of Szegö. One version of this theorem [17], gives the asymptotic behaviour of a determinant $D_{n-1}(f):=\operatorname{det}\left(b_{\mu \nu}\right)_{0 \leqslant \mu, \nu \leqslant n-1}$ whose generic element can be expressed as

$$
b_{\mu \nu}=\int_{-1}^{1} x^{\mu+\nu} f(x) \mathrm{d} x
$$

that is to say, the entries of the determinant can be expressed as the moments of a function $f(x)$.

The theorem says that, as $n \rightarrow \infty$,

$$
D_{n-1}(f)=D_{n-1}(1) \exp \left(n c_{0}+\frac{1}{8} \sum_{j=1}^{\infty} j c_{j}^{2}+\mathrm{O}(1)\right)
$$


where $c_{k} k=0,1, \ldots$, are the Fourier coefficients of the function $\ln (f(\cos \theta))$ :

$$
c_{k}=\frac{1}{2 \pi} \int_{-\pi}^{\pi} \mathrm{e}^{-\mathrm{i} k \theta} \ln (f(\cos \theta)) \mathrm{d} \theta .
$$

To apply this theorem we need the function $f$. The generic term in the Hankel determinant $\sigma_{q-1}$ can be expressed as an integral as follows:

$$
b_{\mu \nu}=\frac{2}{\pi^{2}} 4^{\mu+v} \int_{-1}^{1} x^{\mu+\nu} K^{\prime}(x) \mathrm{d} x
$$

where $K^{\prime}(x)$ is the complete elliptic integral of the first kind:

$$
K^{\prime}(x)=\int_{0}^{\pi / 2}\left(1-x^{\prime 2} \sin \theta\right)^{-1 / 2} \mathrm{~d} \theta=\frac{\pi}{2}{ }_{2} F_{1}\left(\frac{1}{2}, \frac{1}{2} ; 1 ; x^{\prime 2}\right) .
$$

Here $x^{2}+x^{\prime 2}=1$ and ${ }_{2} F_{1}(a, b ; c ; x)$ is Gauss' hypergeometric series [3].

The function $f$ is then

$$
f(x)=K^{\prime}(x) .
$$

The determinant $D_{n-1}(1)$ can be computed exactly and is given by the following product, using formula (2.2.15) in [27]:

$$
D_{n-1}(1)=2 \prod_{k=1}^{n-1}\left(k+\frac{1}{2}\right)^{-1}\left(\frac{1}{2^{k}} C_{2 k}^{k}\right)^{-2} \text {. }
$$

For large values of $n$

$$
D_{n-1}(1)=2^{-n(n-1)+\mathrm{O}(1)} .
$$
as

Combining with the result of Szegö's theorem, the asymptotic representation of $\sigma_{q-1}$ reads

$$
\sigma_{q-1}=q^{q} \mathrm{e}^{-q+1}\left(\frac{2}{\pi^{2}}\right)^{q} 4^{q(q-1)} D_{q-1}(1) \exp \left(q c_{0}+\frac{1}{8} \sum_{j=1}^{\infty} j c_{j}^{2}+\mathrm{O}(1)\right) .
$$

Here $c_{0}=0.729$.

From this we find, as $q \rightarrow \infty$, for a weak magnetic field $\left(\Phi=2 \pi \frac{1}{q}\right)$,

$$
\prod_{n=1}^{q} \Delta_{n} \sim 2^{-q^{2}} q^{-q} \exp \left(-q d_{0}+d_{1}+\mathrm{O}(1)\right)
$$

where $d_{0}$ and $d_{1}$ are constants which cannot be made precise because of the inequality we started with, equation (2.6), and because of the approximations we have used, equations (5.1) and (5.4).

Suppose now that the bandwidths are written as an exponential:

$$
\Delta_{n}=\mathrm{e}^{-\mu_{n} q} .
$$

From the above definition we obtain the asymptotic formula for the average value $\mu_{n}$ :

$$
\frac{\sum_{n=1}^{q} \mu_{n}}{q}=\ln 2+\frac{\ln q}{q}-\frac{d_{0}}{q}+\mathrm{O}\left(\frac{1}{q}\right) \text {. }
$$

At the limit,

$$
\langle\mu\rangle:=\lim _{q \rightarrow \infty} \frac{\sum_{n=1}^{q} \mu_{n}}{q}=\ln 2 .
$$




\section{Conclusions}

This paper contains asymptotic results on the bandwidths in the Hofstadter spectrum for twodimensional electrons in a magnetic field. Besides definite results, it also leaves some open questions. The important results are: the asymptotic formula (5.14), the formula for the edge of the spectrum (4.4) and the conjecture on the general formula for the eigenvalue moments (3.15).

The open questions are. What is the general formula for the polynomials $P_{m}(k)$ ? How do we obtain the fractal structure of the edge of the spectrum from the eigenvalue moments? How do we find the asymptotic representation for the product of the bandwidths for every flux, using Szegö's formula?

Papers $[1,4,5,11-16,18,19,22,23,28,31,32]$ are included as useful general references for this subject.

\section{Acknowledgments}

I am grateful to P B Wiegmann for introducing me to this subject and for his many valuable comments and ideas. I would like to express my gratitude to B Simon for helpful discussions and for the fruitful scientific environment he created during the time this paper was written. I owe special thanks to A G Abanov, P Di Francesco, Y Last, V Sahakian, A Soshnikov and J Talstra, for stimulating discussions.

\section{Appendix}

Here the first eight polynomials $P_{m}(k)$ are listed, which appeared in the eigenvalue moment formula (3.15). The following formulae are conjectured from the first 25 eigenvalue moments (3.14):

$$
\begin{aligned}
& P_{1}=-3^{-1} \\
& P_{2}=90^{-1}\left(7 k^{2}-4 k-15\right) \\
& P_{3}=-1890^{-1}\left(31 k^{4}-60 k^{3}-101 k^{2}+66 k+280\right) \\
& P_{4}=37800^{-1}\left(127 k^{7}-1044 k^{6}+2246 k^{5}+328 k^{4}+7 k^{3}-12244 k^{2}-220 k+25200\right) \\
& P_{5}=-3742200^{-1}\left(2555 k^{9}-31887 k^{8}+137946 k^{7}-243774 k^{6}+290499 k^{5}-796527 k^{4}\right. \\
& \left.\quad+647416 k^{3}+1661436 k^{2}-95184 k-3991680\right) \\
& P_{6}=10216206000^{-1}\left(1414477 k^{12}-32155043 k^{11}+300761927 k^{10}\right. \\
& \quad-1517115007 k^{9}+4720753473 k^{8}-10693488621 k^{7}+20222071853 k^{6} \\
& \quad-25923159133 k^{5}+11996284390 k^{4}+11841173876 k^{3} \\
& \left.\quad+45697115160 k^{2}+12156530400 k-90810720000\right) \\
& \quad-819736201 k^{11}+3945073115 k^{10}-13604875839 k^{9} \\
& \quad+36023850727 k^{8}-73323185763 k^{7}+108429092220 k^{6}
\end{aligned}
$$




$$
\begin{aligned}
& -120643403168 k^{5}+122711359264 k^{4}-65709283536 k^{3} \\
& \left.-63479070720 k^{2}-19852750080 k+186810624000\right)
\end{aligned}
$$

$$
\begin{aligned}
P_{8}=20841060 & 240000^{-1}\left(118518239 k^{17}-5242493627 k^{16}+104055035258 k^{15}\right. \\
& -1233596237660 k^{14}+9844400576738 k^{13}-56735776354034 k^{12} \\
& +247874192827336 k^{11}-847184326535620 k^{10} \\
+ & 2294971824780007 k^{9}-4924658969019451 k^{8} \\
& +8359347851375254 k^{7}-11207926976651080 k^{6} \\
& +11409224332806816 k^{5}-7994766402003888 k^{4} \\
& +4489556615965152 k^{3}-3442931101232640 k^{2} \\
& -806985556876800 k+4668397493760000) .
\end{aligned}
$$

\section{References}

[1] Abanov A G, Talstra J C and Wiegmann P B 1998 Asymptotically exact wave functions of the Harper equation Phys. Rev. Lett. 81 2112-5

[2] Abanov A G, Talstra J C and Wiegmann P B 1998 Hierarchical structure of Azbel-Hofstadter problem: strings and loose ends of Bethe ansatz Nucl. Phys. B 525 571-96

[3] Abramowitz M and Stegun I A (ed) 1964 Handbook of Mathematical Functions (NBS Appl. Math. Ser. vol 55) (Washington, DC: US Govt Printing Office)

[4] Avron J, van Mouche P and Simon B 1990 On the measure of the spectrum for the almost Mathieu operator Commun. Math. Phys. 132 103-18

[5] Bellissard J and Simon B 1982 Cantor spectrum for the almost Mathieu equation J. Funct. Anal. $48408-19$

[6] Barelli A and Fleckinger R 1992 Phys. Rev. B 4611 559-69

[7] Bellissard J and Barelli A 1993 J. Physique 3 471-99

[8] Bellissard J, Camacho C J, Barelli A and Claro F 1997 Exact random walk distributions using noncommutative geometry J. Phys. A: Math. Gen. 30 L707-9

[9] Bilingsley P 1968 Convergence of Probability Measures (New York: Wiley)

[10] Chambers W G 1965 Linear-network model for magnetic breakdown in two dimensions Phys. Rev. 140 A135-43

[11] Guillement J P, Helffer B and Treton P 1989 Walk inside Hofstadter's butterfly J. Physique 50 2019-58

[12] Harper P G 1955 Single band motion of conduction electrons in a uniform magnetic field Proc. Phys. Soc. A 68 874

[13] Helffer B and Sjöstrand J 1988 Analyse semi-classique pour léquation de Harper I (avec application á létude de léquation de Schrödinger avec champ magnétique) Suppl. Bull. Soc. Math. France vol 116, Fasc. 4, Mémoire 34

[14] Hiramoto H and Kohomoto M 1992 Electronic spectral and wavefunction properties of one-dimensional quasiperiodic systems: a scaling approach Int. J. Mod. Phys. B 6 281-320

[15] Hofstadter D 1976 The energy-levels of Bloch electrons in rational and irrational magnetic fields Phys. Rev. B $142239-49$

[16] Jitomirskaya S and Last Y 1998 Anderson localization for the almost Mathieu equation. III. Semiuniform localization, continuity of gaps and measure of the spectrum Commun. Math. Phys. 195 1-14

[17] Johansson K 1988 On Szegö's asymptotic formula for toeplitz determinants and generalizations Bull. Sci. Math. II 112 257-304

[18] Krasovsky I V 1999 Bethe ansatz for the Harper equation: solution for a small commensurability parameter Phys. Rev. B 59 322-8

[19] Hoshi K and Hatsugai Y 2000 Landau levels from the Bethe ansatz equations Phys. Rev. B 61 4409-12

[20] Kreft C 1995 Spectral analysis of Hofstadter-like models Thesis Technischen Universität Berlin, Berlin

[21] Last Y 1994 Zero measure spectrum for the almost Mathieu operator Commun. Math. Phys. 164 421-32

[22] Last Y 1995 Almost everything about the almost Mathieu operator. I Proc. 11th Int. Conf. of Mathematical Physics ed D Iagolnitzer (Boston, MA: Academic) pp 366-72

[23] Last Y and Wilkinson M 1992 A sum rule for the dispersion relations of the rational Harper equation J. Phys. A: Math. Gen. 25 6123-33 
[24] Lévy P 1951 Wiener's random function and other Laplacian random functions ed J Neyman Proc. 2nd Berkeley Symp. on Mathematical Statistics and Probability (Berkeley, CA: University of California Press) pp 171-87

[25] Mingo J A and Nica A 1998 On the distribution of the area enclosed by a random walk on $Z^{2}$ J. Comb. Theory series A 84 55-86

[26] Rammal R and Bellissard J 1990 An algebraic semiclassical approach to Bloch electrons in a magnetic field $J$. Physique 51 1803-30

[27] Szegö G 1939 Orthogonal Polynomials (American Mathematical Society Colloquium Publications, vol XXIII) (New York: American Mathematical Society)

[28] Thouless D J 1990 Scaling for the discrete Mathieu equation Commun. Math. Phys. 127 187-93

[29] Wannier G H, Obermair G M and Ray R 1979 Magnetoelectronic density of states for a model crystal Phys. Status Solidi (b) 93337

[30] Wiegmann P B and Zabrodin A V 1994 Quantum group and magnetic translations. Bethe-ansatz for the AsbelHofstadter problem Nucl. Phys. B 422 495-514

[31] Wilkinson M 1987 An exact renormalization group for Bloch electrons in a magnetic field J. Phys. A: Math. Gen. 201791

[32] Yong Tan and Thouless D J 1991 Total bandwidth for the Harper equation III. Corrections to scaling J. Phys. A Math. Gen. 24 4055-66 